\title{
BMJ Open Prevalence, incidence and associated mortality of cardiovascular disease in patients with chronic kidney disease in low- and middle-income countries: a protocol for a systematic review and meta-analysis
}

\author{
Mazou N Temgoua, ${ }^{1}$ Celestin Danwang, ${ }^{2}$ Valirie Ndip Agbor, ${ }^{3}$ \\ Jean Jacques Noubiap ${ }^{4}$
}

To cite: Temgoua MN, Danwang C, Agbor VN, et al. Prevalence, incidence and associated mortality of cardiovascular disease in patients with chronic kidney disease in low- and middleincome countries: a protocol for a systematic review and meta-analysis. BMJ Open 2017;7:e016412. doi:10.1136/ bmjopen-2017-016412

- Prepublication history for this paper is available online. To view these files, please visit the journal online (http://dx.doi org/10.1136/bmjopen-2017016412).

Received 12 February 2017 Revised 29 June 2017 Accepted 7 July 2017

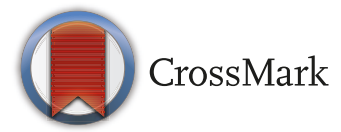

For numbered affiliations see end of article.

Correspondence to Dr Jean Jacques Noubiap; noubiapjj@yahoo.fr

\section{ABSTRACT}

Introduction Chronic kidney disease (CKD) is a global public health problem, with cardiovascular disease (CVD) being the major cause of mortality in these patients. Despite a high burden of CKD among patients in low/ middle-income countries (LMICs), evidence on the distribution of CVD among these patients is lacking. This review seeks to determine the prevalence, incidence and mortality risks of CVD in patients with CKD in LMICs. Methods and analysis A systematic search of Medline, Scopus, Embase, Cumulative Index of Nursing and Allied Health and WHO Global Health Library databases for published studies reporting on the prevalence, incidence and associated mortality risk of CVD in CKD patients in LMICs will be conducted from 1 May 1987 to 1 July 2017 with no language restriction. Two authors will independently screen, select studies, extract data and assess the risk of bias in each study. Clinically homogeneous studies will be pooled after assessing for clinical and statistical heterogeneity using the $\chi^{2}$ test on Cochrane's $Q$ statistic which is quantified by $I^{2}$ values; assuming that $I^{2}$ values of $25 \%, 50 \%$ and $75 \%$ represent low, medium and high heterogeneity, respectively. Funnelplot analysis and Egger's test will be used to detect publication bias. Results will be presented according to WHO Regions (Africa, Americas, Eastern Mediterranean, Europe, South-East Asia and Western Pacific).

Ethics and dissemination This proposed study will not require ethical approval as it will be based on published data. We will publish the final report of this review in a peer-reviewed journal, and the findings will be disseminated to the appropriate health authorities.

\section{INTRODUCTION}

Chronic kidney disease (CKD) is a public health problem worldwide with a global prevalence of $11 \%$ to $13 \% .^{1}$ Hypertension and diabetes remain the major risk factors for $\mathrm{CKD}^{2}{ }^{2}$ According to the 2010 Global Burden of Disease study, CKD ranked 27th in the list
Strengths and limitations of this study

- To the best of our knowledge, this will be the first review on cardiovascular disease in patients with chronic kidney disease in low/middle-income countries (LMICs); result will help to understand the burden of this disease and put in place cost effective diagnostic and management strategy.

- We will use powerful meta-analysis techniques to determine accurate estimates.

- A major limit of this review could be the few number of good quality studies in LMICs.

- Another possible limitation may be various definition criteria of cardiovascular disease (CVD) in studies done on the topic in LMICs.

- It is often unclear if CVD precedes chronic kidney disease or vice versa. This therefore represents a potential limitation to this study.

of causes of total number of deaths worldwide in 1990 rose to 18 th in $2010 .{ }^{3}$ Low/middle-income countries (LMICs) have the greatest burden of CKD, accounting for $80 \%$ of all cases of CKD globally. ${ }^{4}$ The prevalence of CKD in LMICs varies between $14.3 \%$ and $36.1 \%,{ }^{5}$ with an annual incidence of end-stage renal disease of over 500000 patients, resulting in premature death in a vast majority cases. ${ }^{6}$ The epidemiology of cardiac disease in CKD is well documented in high-income countries (HIC) robust and appropriate diagnostic and therapeutic techniques are available. ${ }^{7}$ Fifteen per cent of patients who start dialysis therapy have a left ventricular systolic dysfunction. ${ }^{8}$ Also, advanced age, anaemia, hypertension and coronary heart disease represent the main causes of myocardial dysfunction in patients with end-stage renal disease. ${ }^{8}$ 
The occurrence of congestive heart failure, cerebrovascular disease, atrial fibrillation, peripheral arterial disease and sudden cardiac death in patients with CKD is high. ${ }^{9}$ Nevertheless, cardiovascular disease (CVD) remains underdiagnosed and undertreated among patients with CKD in LMICs. ${ }^{10}$ In this part of the world, the burden of CKD is high due to lack of resources and late presentation of patients to the nephrologist. ${ }^{11}$ Even though the morbidity and mortality of CKD in LMICs is high, ${ }^{4}$ accurate epidemiological data on CVD in patients with CKD are still unavailable in these settings. An appropriate knowledge on the occurrence of CVD in patients with CKD will help policy-makers in LMICs to allocate the necessary resources aimed at curbing the burden of CVD in CKD patients. This systematic review and meta-analysis seeks to summarise available data on the prevalence, incidence and mortality risk of major forms of CVD like: left ventricular systolic dysfunction, coronary artery disease, congestive heart failure, cerebrovascular disease, atrial fibrillation, peripheral arterial disease and sudden cardiac death in patients with CKD in LMICs.

\section{Objective}

We aim to conduct a systematic review and meta-analysis to determine the prevalence, incidence and mortality risk of CVD in patients with CKD in LMICs.

\section{Review question}

This review of studies published in the past 30 years will endeavour to respond to the following questions:

1. What is the prevalence of CVD in patients with CKD in LMICs?

2. What are the cumulative incidence (number of new cases per total number of participants at risk) and the incidence rate (incidence per total person-years or person-months at risk) of CVD in patients with CKD in LMICs?

3. What is the mortality risk associated with CVD in patients with CKD in LMICs?

\section{METHODS AND ANALYSIS}

\section{Criteria for considering studies for the review}

Inclusion criteria

All studies on human subjects with the following criteria will be included:

1. Observational studies ((cross-sectional, case-control and cohort studies)) with available data on the incidence or mortality risk of CVD in CKD in LMICs;

2. Adult participants (age $\geq 18$ years) residing LMICs;

3. Duration: We will consider all published or unpublished data from 1 May 1987 to 1 July 2017;

4. Language restriction: no language restriction.

\section{Exclusion criteria}

We will not consider:

1. Studies conducted among populations of LMICs origin residing outside these countries;
2. Letters, reviews, commentaries, editorials and case series;

3. Studies lacking data to compute the prevalence, incidence or mortality of CVD in patients with CKD;

4. Studies without an explicit method description;

5. Duplicates studies: the most comprehensive, most recent and with the largest sample size will be considered;

6. Studies whose full data will not be accessible even after request from the authors;

7. Studies which relied self-reporting as criteria for identification of CVD.

\section{Source of information}

This systematic review will be reported according to the Meta-Analysis of Observational Studies in Epidemiology Guidelines. ${ }^{12}$

\section{Search strategy for identifying relevant studies}

The search strategy will be implemented in two stages.

\section{Bibliographic database searches}

First, relevant abstracts published on the prevalence, incidence and mortality of CVD in CKD in LMICs will be identified by searching Medline, Scopus, Embase, Cumulative Index of Nursing and Allied Health (CINAHL) and WHO Global Health Library databases from 1 May 1987 to 1 July 2017 , using text words and medical subject heading terms. Example of key search terms include: 'chronic kidney disease' or 'chronic renal failure' or 'chronic kidney injury' and 'cardiovascular disease' or 'cardiac disease' or 'heart disease'. Also, we will include all cardiovascular disease reported in previous studies such as: coronary heart disease or myocardial infarction, cerebrovascular disease or stroke, congestive heart failure, atrial fibrillation, peripheral arterial disease and sudden cardiac death. The names of all LMICs will then be added to the search. Finally, we shall search relevant conference papers from the World Congress of Nephrology and the African Nephrology congresses. The main search strategy for PubMed is shown in table 1. Subsequently, the abstracts of all eligible papers will be reviewed and full articles will be accessed through Medline, HINARI, CINAHL or journals' websites.

\section{Searching for others sources}

References of all relevant original and review articles will be searched for additional potential data sources, and their full texts will be accessed in a similar way. Those authors whose full-text papers will not be accessible by the numerous internet-based sources will be directly contacted via email to provide them. In case of no feedback from these authors, the corresponding studies will be excluded.

\section{Selection of studies for inclusion in the review}

The titles and abstracts of paper obtained from the searches will be screened and the full texts of potentially 


\begin{tabular}{|c|c|}
\hline Search & Search terms \\
\hline$\# 1$ & $\begin{array}{l}\text { 'Chronic kidney disease' OR 'Chronic renal failure' OR ‘Chronic kidney insufficiency' OR 'Chronic renal } \\
\text { insufficiency' OR 'Chronic kidney failure' OR 'End-Stage Renal Disease' OR 'end/target-renal damage' OR } \\
\text { nephropathy OR ‘proteinuria' OR 'dialysis-dependent' }\end{array}$ \\
\hline \#2 & $\begin{array}{l}\text { 'Cardiovascular disease' OR ‘Cardiac disease' OR ‘Cardiovascular event' OR Heart disease OR 'Coronary heart } \\
\text { disease' OR 'Myocardial infarction' OR 'Myocardial ischemia' OR ‘Cerebrovascular disease' OR Stroke OR 'Brain } \\
\text { ischemia' OR ‘Cerebral hemorrhage' OR 'Heart failure' OR 'Cardiac failure' OR ‘Congestive heart failure' OR } \\
\text { 'Ventricular dysfunction Heart failure' OR 'Atrial Fibrillation' Or 'Cardiac arrhythmia' OR 'Peripheral arterial disease' } \\
\text { OR ‘Intermittent claudication' OR 'Sudden cardiac death' Or ‘Sudden cardiac arrest' }\end{array}$ \\
\hline$\# 3$ & 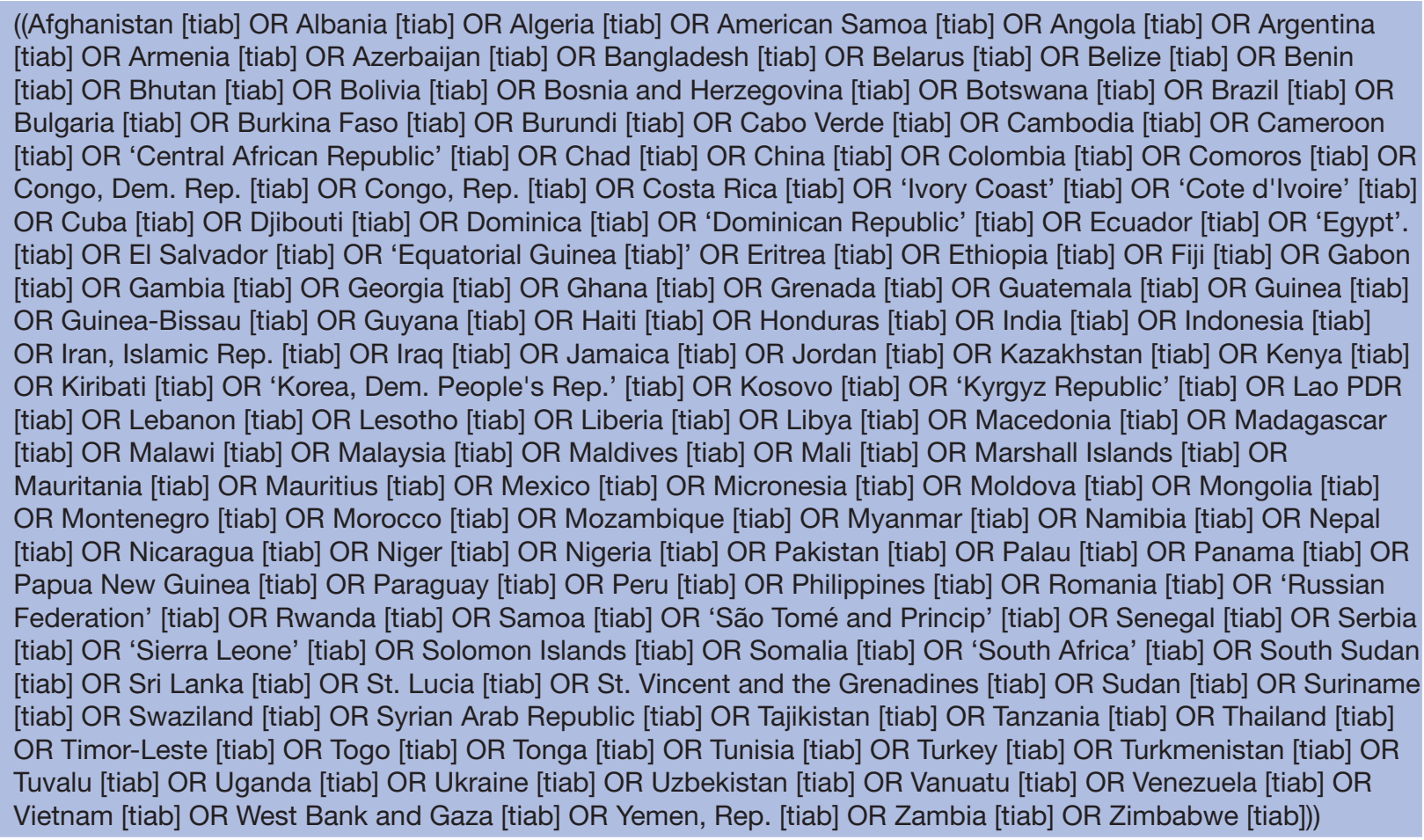 \\
\hline \#4 & \#1 AND \#2 AND \#3 \\
\hline \#5 & \# 4 Limits: $1987 / 05 / 01$ to $2017 / 07 / 01$ \\
\hline
\end{tabular}

eligible papers will be retrieved by two authors (MNT and CD). Thereafter, they will independently review the full texts of the retrieved papers, compare their results and resolve any discrepancy by discussion and consensus. If a decision cannot be reached by both authors after discussion, this will be solved by a third author (VNA). Figure 1 depicts the procedure that will be followed in selecting studies for this review.

\section{Assessment of methodological quality and reporting of data}

The quality of eligible studies will be assessed by two authors (MNT and CD) using an adapted version of the Risk of Bias Tool developed by Hoy et al. ${ }^{13}$ The risk of selection and attrition bias will be assessed using the Cochrane guidelines available in Review Manager V.5.3 (http://tech.cochrane.org/revman). Furthermore, the reporting quality of each study will be assessed using the Strengthening the Reporting of Observational Studies in Epidemiology checklist. ${ }^{12}$

\section{Data extraction and management}

A data extraction sheet will be used to collect information about the author, the country, the year of publication, the study design, the sample size, the mean or median age of the population, the sex ratio, the stage of CKD, prevalence, cumulative incidence of each form of CVD, incidence rate and mortality risk of CVD in CKD. In studies where information is lacking, we will directly contact the corresponding author to request the information. Where appropriate, results will be disaggregated for multinational studies to show necessary data of individual countries. Otherwise, it will be presented as one and the countries in which the studies were done will be listed.

\section{Data synthesis and analysis}

Data collected will be analysed using Stata software V.13 (Stata Corp). A meta-analysis will be conducted for data obtained from similar studies (same CVD). SEs for the study-specific estimates will be determined from the point estimate (mean, proportion, OR or relative risks) 


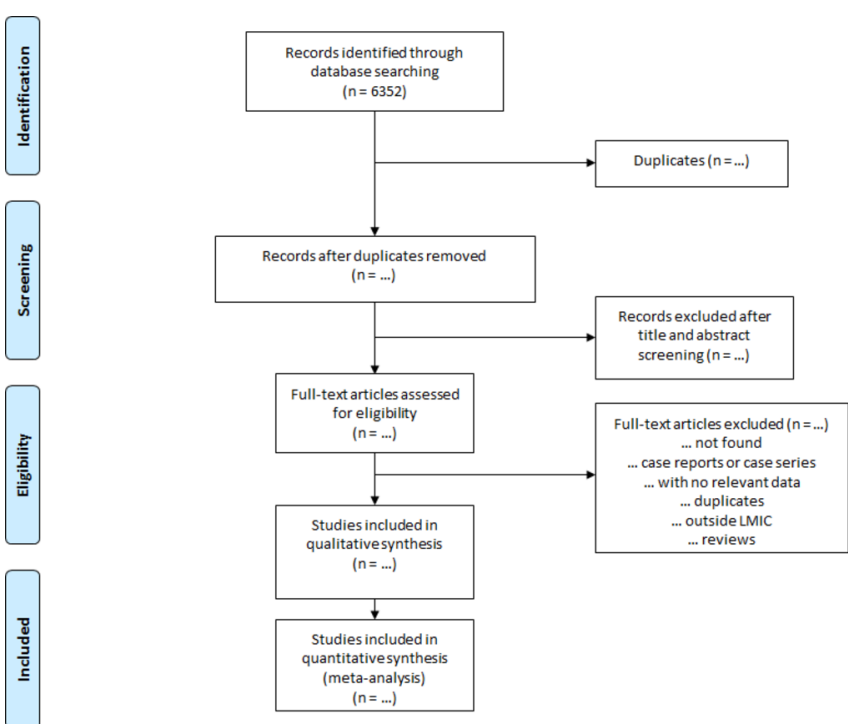

Figure 1 Flow diagram for study selection. LMIC, low/ middle-income country.

and their upper and lower CIs, assuming a binomial distribution. A random effect meta-analysis model will be used to pool the study-specific estimates to obtain overall summary of the incidence and mortality rate across studies. The Freeman-Tukey double arc-sine transformation will be use to stabilise variance of individual studies ${ }^{14}$ and therefore keep the effect of studies with extremely small or large estimates. Heterogeneity will be assessed using the $\chi^{2}$ test on Cochrane's $Q$ statistic ${ }^{15}$ which is quantified by $\mathrm{I}^{2}$ values, assuming that $\mathrm{I}^{2}$ values of $25 \%, 50 \%$ and $75 \%$ represent low, medium and high heterogeneity, respectively. ${ }^{16}$ Where high heterogeneity will be detected, we will perform a subgroup analysis and meta-regression to detect its possible sources using the following grouping variables: age groups, sex, study setting (rural vs urban; hospital vs community based), country and geographical region (Africa, Americas, Eastern Mediterranean, Europe, South-East Asia and Western Pacific), type of CVD, stage of CKD and study quality. The difference between groups will be considered if $p$ value $<0.05$. We will assess the presence of publication bias using funnel plots and Egger's test. ${ }^{17}$ Publication bias will be confirmed if $\mathrm{p}$ value on Egger's test $<0.10$. The Cohen's $\kappa$ coefficient ${ }^{18}$ will be used to assess the inter-rater agreement for study inclusion.

\section{Presentation and reporting of results}

A flow diagram will be used to demonstrate the study selection process and reasons for exclusion of studies will be detailed. Summary tables and forest plots will be used to present quantitative variables where appropriate. Quality scores and risk of bias for each eligible study will be reported using tabulations and narrative summaries.

Author affiliations

${ }^{1}$ Department of Internal Medicine and Specialties, Faculty of Medicine and Biomedical Sciences, University of Yaoundé I, Yaoundé, Cameroon
${ }^{2}$ Department of Surgery and Specialties, Faculty of Medicine and Biomedical Sciences,University of Yaoundé I, Yaoundé, Cameroon

${ }^{3}$ Ibal sub-Divisional Hospital, Oku, North-west Region, Cameroon

${ }^{4}$ Department of Medicine, Groote Schuur Hospital and University of Cape Town, Cape Town, South africa

Contributors MNT and JJN conceived and designed the protocol. MNT drafted the manuscript. CD, VNA and JJN critically revised the manuscript for intellectual content. JJNN is the guarantor of the review. All authors approved the final version of the manuscript.

Competing interests None declared.

Patient consent Obtained.

Provenance and peer review Not commissioned; externally peer reviewed.

Open Access This is an Open Access article distributed in accordance with the Creative Commons Attribution Non Commercial (CC BY-NC 4.0) license, which permits others to distribute, remix, adapt, build upon this work non-commercially, and license their derivative works on different terms, provided the original work is properly cited and the use is non-commercial. See: http://creativecommons.org/ licenses/by-nc/4.0/

(c) Article author(s) (or their employer(s) unless otherwise stated in the text of the article) 2017. All rights reserved. No commercial use is permitted unless otherwise expressly granted.

\section{REFERENCES}

1. Hill NR, Fatoba ST, Oke JL, et al. Global prevalence of chronic kidney disease-a systematic review and meta-analysis. PLoS One 2016;11:e0158765.

2. Chronic kidney disease - World Kidney Day. http://www. worldkidneyday.org/faqs/chronic-kidney-disease/

3. Global facts: about kidney disease-The National Kidney Foundation. https://www.kidney.org/kidneydisease/global-factsabout-kidney-disease

4. Stanifer JW, Muiru A, Jafar TH, et al. Chronic kidney disease in low- and middle-income countries. Nephrol Dial Transplant 2016;31:868-74.

5. Ene-lordache B, Perico N, Bikbov B, et al. Chronic kidney disease and cardiovascular risk in six regions of the world (ISN-KDDC): a cross-sectional study. Lancet Glob Health 2016;4:e307-e319.

6. Ojo A. Addressing the global burden of chronic kidney disease through clinical and translational research. Trans Am Clin Climatol Assoc 2014;125:229-46.

7. Gansevoort RT, Correa-Rotter R, Hemmelgarn BR, et al. Chronic kidney disease and cardiovascular risk: epidemiology, mechanisms, and prevention. Lancet 2013;382:339-52.

8. Lisowska A, Musiał WJ. Heart failure in patients with chronic kidney disease. Rocz Akad Med Bialymst 2004;49:162-5.

9. Herzog CA, Asinger RW, Berger AK, et al. Cardiovascular disease in chronic kidney disease. A clinical update from kidney disease: improving global outcomes (KDIGO). Kidney Int 2011;80:572-86.

10. Said S, Hernandez GT. The link between chronic kidney disease and cardiovascular disease. J Nephropathol 2014;3:99-104.

11. Lee J, Lee JP, An JN, Jn A, et al. Factors affecting the referral time to nephrologists in patients with chronic kidney disease. Medicine 2016;95:e3648.

12. Stroup DF, Berlin JA, Morton SC, et al. Meta-analysis of observational studies in epidemiology: a proposal for reporting. Meta-analysis of observational studies in Epidemiology (MOOSE) group. JAMA 2000;283:2008-12.

13. Hoy $D$, Brooks $P$, Woolf $A$, et al. Assessing risk of bias in prevalence studies: modification of an existing tool and evidence of interrater agreement. J Clin Epidemiol 2012;65:934-9.

14. Miller JJ. The inverse of the freeman-Tukey double arcsine transformation. Am Stat 1978;32:138.

15. Kulinskaya E, Dollinger MB. An accurate test for homogeneity of odds ratios based on Cochran's Q-statistic. BMC Med Res Methodol 2015;15:49.

16. Higgins JP, Thompson SG. Quantifying heterogeneity in a metaanalysis. Stat Med 2002;21:1539-58.

17. Egger M, Davey Smith G, Schneider M, et al. Bias in meta-analysis detected by a simple, graphical test. BMJ 1997;315:629-34.

18. McHugh ML. Interrater reliability: the kappa statistic. Biochem Med 2012;22:276-82. 